УДК 621.01

ВАК 05.02.18

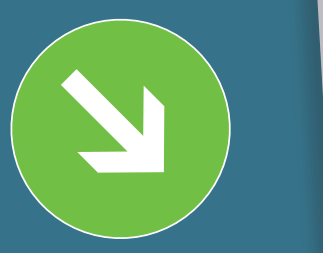

Ключевые слова:

Число степеней

свободы, кони-

ческая передача,

ременная передача,

выходное звено

\title{
ОПРЕДЕЛЕНИЕ ЧИСЛА СТЕПЕНЕЙ СВОБОДЫ МЕХАНИЗМОВ С ПОСТОЯННОЙ ТОЧКОЙ ВВОДА ИНСТРУМЕНТА
}

\author{
Роберт ЧЕРНЕЦОВ, ЕВГеНИЙ ВЕЛИЕВ, ВИктор ГЛАЗУНОВ, \\ Сергей СКВОРЦОВ, Наталья КОВАЛЕВА
}

Приведены два варианта механизмов, обеспечивающих постоянство положения точки ввода инструмента, основанных на конической и ременной передаче, выходное звено которых совершает сферическое движение. Определено число степеней свободы для каждого механизма.

Для многих задач современной техники необходимы устройства, в которых должно быть обеспечено постоянство положения точки ввода инструмента в рабочую зону. Речь идет, в частности, об измерительных устройствах, предназначенных для исследования свойств плазмы [1]. Такое же условие необходимо выполнить при разработке манипуляционных механизмов, предназначенных для хирургических операций [2].

Одним из решений данной проблемы является механизм, основанный на применении шарнирных параллелограммов [3]. При этом угол поворота привода равен углу поворота выходного звена.

В данной статье представлено два новых механизма, решающих подобную задачу. Один из них содержит конические передачи, в другом имеют место ременные передачи. В обоих случаях приводы, определяющие ориентацию выходного звена, расположены вне рабочей зоны. Конические и ременные передачи служат для сохранения постоянства положения точки ввода инструмента. Указанные устройства можно рассматривать как механизмы параллельной структуры. Как известно, механизм параллельной структуры имеет высокие показатели точности и нагрузочной способности [4-10]. Эти качества обусловлены тем, что указанные механизмы воспринимают нагрузку подобно пространственным фермам. Однако, в данном случае наличие механизмов параллельной структуры позволяет решить задачу создания устройств, обеспечивающих постоянную точку ввода инструмента.

\section{РЕШЕНИЕ ЗАДАЧИ ПО ОПРЕДЕЛЕНИЮ СТЕПЕНЕЙ СВОБОДЫ ДЛЯ МЕХАНИЗМА С КОНИЧЕСКОЙ ПЕРЕДАЧЕЙ}

Механизм, представленный на рис. 1 , состоит из основания 1 , выходного звена 2, рабочего органа 3 , поворотной платформы 6, которая приводится во вращение двигателем 5 через вращательную кинематическую пару 4. С поворотной платформой 6 через кинематическую пару 7, приводимую в движение двигателем 8, связано звено 9, которое через кинематическую вращательную пару 10 связано с промежуточным звеном 11. Данное звено сопряжено через кинематическую пару 12 с выходным звеном 2. Для того чтобы обеспечить постоянство передаточных отношений между двигателем 8 и выходным звеном 2 , механизм снабжен двумя коническими передачами, при этом коническое колесо 13 жестко связано с начальным звеном 9. Конические колеса 14 и 16 расположены на одном валу 15, при этом колесо 16 сопряжено с коническим колесом 17, жестко связанным спромежуточным звеном 11. Так же с указанным звеном 11 жестко связано коническое колесо 18, сопряженное с коническим колесом 19 , которое расположено на валу 20 вместе с коническим колесом 21 . Указанное колесо 21 сопряжено с коническим колесом 22, жестко связанным с выходным звеном 2.

Наличие конических передач обеспечивает равные углы поворота начального звена 9 и выходного звена 2.

Рабочий орган 3 относительно выходного звена 2 может перемещаться поступательно под действием линейного двигателя 24 и вращательно под действием 


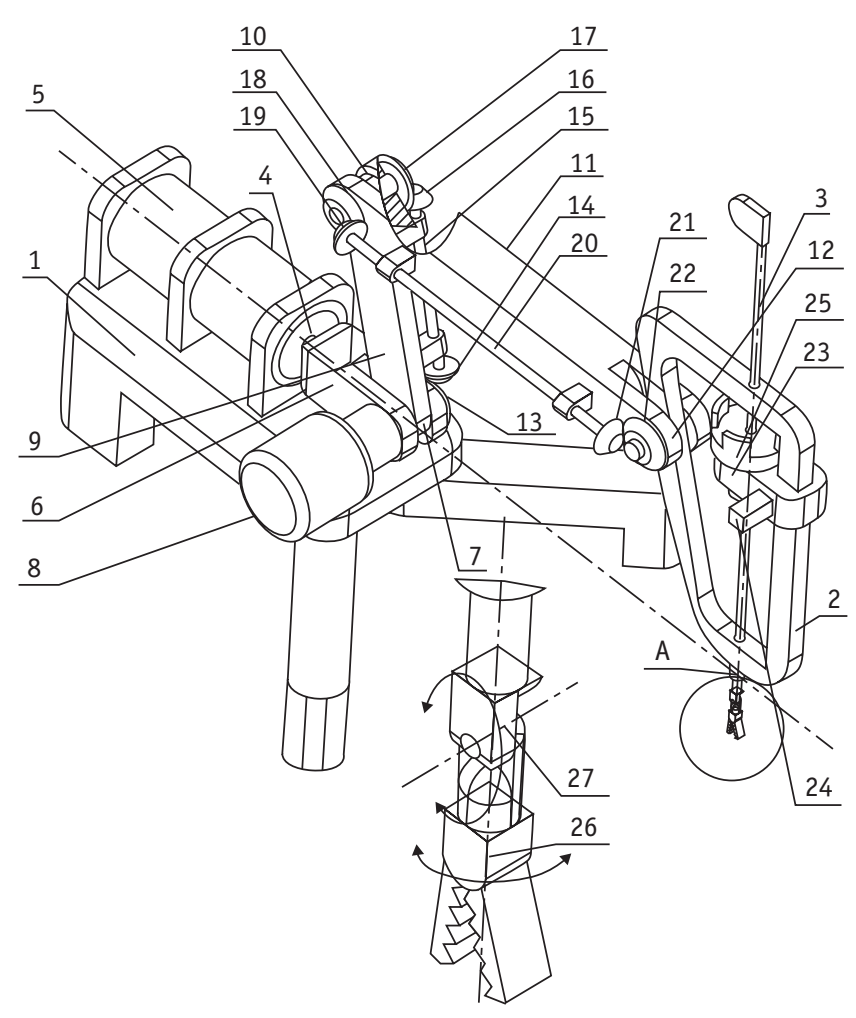

Puc. 1. Механизм с конической передачей

двигателя 23. Ось рабочего органа 3 пересекает продолжение оси двигателя 5 в точке А, положение которой неизменно. С рабочим органом 3 сопряжено устройство фиксации 25 , обеспечивающее быструю смену инструмента, который через вращательные кинематические пары 26 и 27 связан с рабочим органом 3.

Основание 1 с вращательным двигателем 5, поворачивающим подвижную платформу 6 с расположенными на ней узлами манипулятора, образует первую кинематическую пару пятого класса. Вал двигателя 8 связан с одним концом звена 9 и поворачивает входное звено вокруг своей оси, образуя вторую кинематическую пару пятого класса.

Конические колеса 13 и 17, сопряженные с коническими колесами 14 и 16, установленными соосно на вращательном валу 15, образующие первую и вторую пару первого класса, формируют первую зубчатую передачу, обеспечивающую параллельность оси звена 11 и оси привода 5 при повороте звена 9 вращательным двигателем 8. Звенья 9 и 11 образуют третью кинематическую пару пятого класса. На противоположном от двигателя 8 конце звена 9 жестко закреплено коническое зубчатое колесо 18 , установленное соосно на одном валу с зубчатым коническим колесом 17 , образующим четвертую пару пятого класса и сопряженно с коническим зубчатым колесом 19, образующим третью пару первого класса, установленным соосно на одном вращательном валу 20 с коническим зубчатым колесом 21 , сопряженным с коническим колесом 22, установленным на выходном звене 2 , образующие четвертую пару первого класса, формируют вторую зубчатую передачу. Коническое колесо 22 и выходное звено 2 образуют пятую пару пятого класса, а звено 11 и выходное звено 2 образуют шестую пару пятого класса. Вторая зубчатая передача обеспечивает параллельность звена 9 и рабочего органа 3. Рабочий орган 3 связан с выходным звеном 2 посредством пары четвертого класса.

Определяем количество степеней свободы механизма по формуле Сомова - Малышева:

$$
W=6 \cdot n-5 \cdot P_{5}-4 \cdot P_{4}-3 \cdot P_{3}-2 \cdot P_{2}-P_{1},
$$

где $n$ - количество промежуточных звеньев цепи, $\mathrm{P}_{5}, \mathrm{P}_{4}, \ldots, \mathrm{P}_{1}$ - количество одно-, двух-, ..., 5-подвижных пар.

Для выходного звена: количество пар 5-го класса $-P_{5}=6$; количество пар 1-го класса $-P_{1}=4$; количество подвижных звеньев кинематической цепи - $n=6$.

Теперь можно рассмотреть структуру данного механизма, используя приведенную выше структурную формулу (1):

$$
W=6 \cdot 6-5 \cdot 6-4=36-30-4=2 .
$$

Таким образом, количество степеней свободы для выходного звена равно 2.

Для рабочего органа: количество пар 5-го класса $-P_{5}=6$; количество пар 4-го класса $-P_{4}=1$; количество пар 1-го класса $-P_{1}=4$; количество подвижных звеньев кинематической цепи $-n=7$. Используя приведенную структурную формулу (1) получаем:

$$
W=6 \cdot 7-5 \cdot 6-4-4=42-30-4-4=4 \text {. }
$$

Количество степеней свободы для рабочего органа равно 4.

Для инструмента: количество пар 5-го класса $P_{5}=6$; количество пар 4-го класса $-P_{4}=2$; количество пар 1-го класса $-P_{1}=4$; количество подвижных звеньев кинематической цепи $-n=8$. Используя приведенную структурную формулу (1) получаем:

$$
W=6 \cdot 8-5 \cdot 6-4 \cdot 2-4=48-30-8-4=6 \text {. }
$$

Количество степеней свободы для инструмента равно 6.

\section{РЕШЕНИЕ ЗАДАЧИ ПО ОПРЕДЕЛЕНИЮ СТЕПЕНЕЙ СВОБОДЫ ДЛЯ МЕХАНИЗМА С РЕМЕННОЙ ПЕРЕДАЧЕЙ}

Механизм, представленный на рис. 2, отличается от рассмотренного выше тем, что вместо конических передач используются ременные передачи. 
Механизм состоит из основания 1 , вращательного двигателя 2, перемещающего платформу 3 , на которой стоит двигатель 4. Выходное звено 5 связано с начальным звеном 6 через промежуточное звено 7. Для обеспечения равенства углов поворота звеньев 5 и 6 применяются ременные передачи, состоящие из шкивов 8 и 9, а также ремня 10 и шкивов 11 и 12, а также ремня 13. Линейный двигатель 14 , а также вращательный двигатель 16 относительно рамки 15 жестко связаны с выходным звеном 5, перемещают рабочий орган 17 , соответственно, вдоль и вокруг его оси. Инструмент связан с рабочим органом посредством вращательных кинематических пар 18 и 19.

Вал двигателя 2 связан с подвижной платформой 3 , образуя первую кинематическую пару пятого класса, на которой размещен вращательный двигатель 4. Оси вращения двигателей 2 и 4 перпендикулярны и пересекаются. Подвижная платформа 3 соединяется с выходным звеном 5 двумя взаимосвязанными кинематическими цепями. Первая кинематическая цепь образована двумя сопряженными звеньями 6 и 7, образующими вторую кинематическую пару пятого класса.

Начало звена 6 неподвижно связано с валом вращательного двигателя 4, образуя третью кинематическую пару пятого класса. Конец звена 7, связанный рамкой 15 с выходным звеном 5, образуют четвертую кинематическую пару пятого класса. Вторая кинематическая цепь, связывающая подвижную платформу 3 с выходным звеном 5, состоит из двух последовательно установленных ременных передач, каждая из которых содержит по два шкива, связанных посредством ремня. Входной шкив 8 первой передачи, неподвижно соединенный с подвижной платформой 3, образуют первую кинематическую пару первого класса, а выходной шкив 9 первой передачи - с началом звена 7, образуют вторую кинематическую пару первого класса. Их связывает ремень 10. Входной шкив 11 второй передачи неподвижно сопряженный с концом звена 6 , образует третью кинематическую пару первого класса. Выходной шкив 12 второй ременной передачи, связанный с возможностью поворота звена 7, образует четвертую кинематическую пару первого класса. Шкивы 11 и 12 второй ременной передачи связаны ремнем 13. На выходном звене 5 установлен линейный двигатель 14, вращательный двигатель 16 и рабочий орган 17 , которые образуют первую кинематическую пару четвертого класса. Ось рабочего органа 17 пересекается с осью вращательного двигателя 2 в точке А, которая сохраняет свое положение при перемещении звеньев манипулятора. Рабочий орган 17 связан с рамкой 15 посредством пары четвертого класса.

Определяем количество степеней свободы механизма по формуле Сомова - Малышева (1).

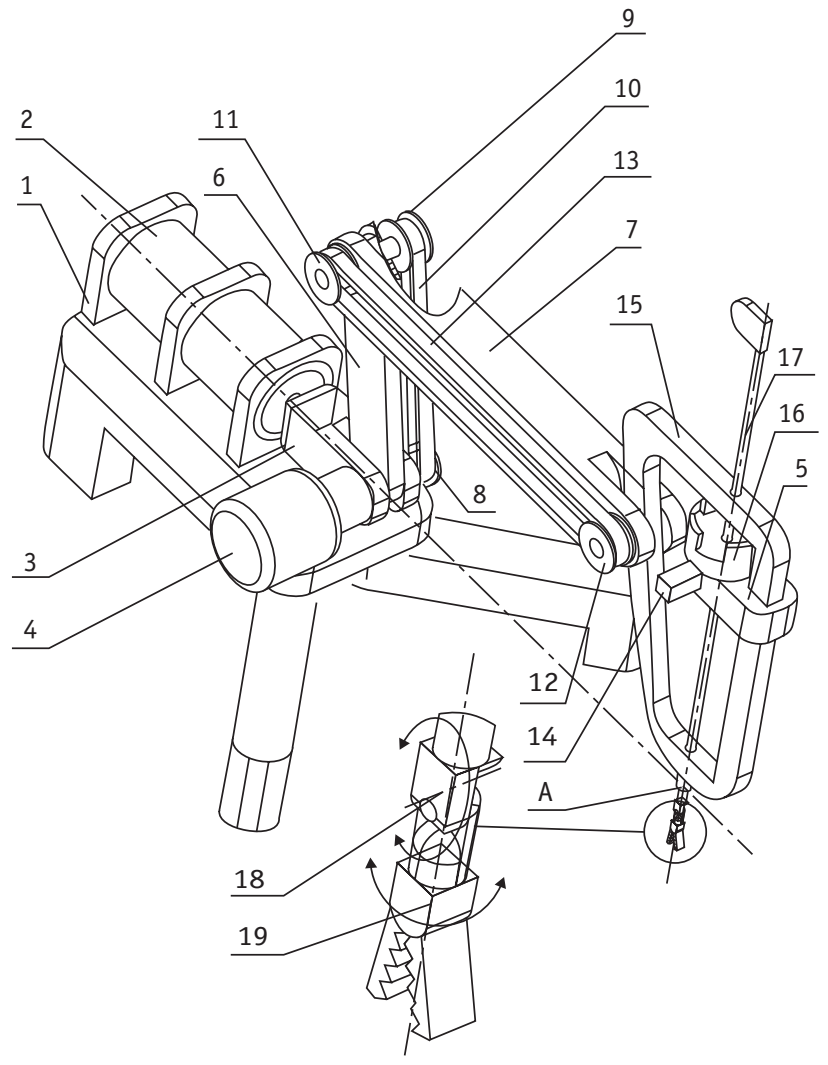

Puc. 2. Механизм с ременной передачей

Для выходного звена: количество пар 5-го класса $-P_{5}=6$; количество пар 1-го класса $-P_{1}=4$; количество подвижных звеньев кинематической цепи $-n=6$.

Теперь можно рассмотреть структуру данного механизма, используя приведенную структурную формулу (1):

$$
W=6 \cdot 6-5 \cdot 6-4=36-30-4=2 .
$$

Таким образом, количество степеней свободы для выходного звена равно 2.

Для рабочего органа; количество пар 5-го класса $-P_{5}=6$; количество пар 4-го класса $-P_{4}=1$; количество пар 1-го класса $-P_{1}=4$; количество подвижных звеньев кинематической цепи - $n=7$.

Используя приведенную структурную формулу (1) получаем:

$$
W=6 \cdot 7-5 \cdot 6-4-4=42-30-4-4=4 .
$$

Количество степеней свободы для рабочего органа равно 4.

Для инструмента: количество пар 5-го класса $P_{5}=6$; количество пар 4-го класса $-P_{4}=2$; количество пар 1-го класса $-P_{1}=4$; количество подвижных звеньев кинематической цепи $-n=8$.

Используя приведенную структурную формулу (1) получаем: 
$W=6 \cdot 8-5 \cdot 6-4 \cdot 2-4=48-30-8-4=6$.

Количество степеней свободы для рабочего органа равно 6.

В данной статье рассмотрены два новых механизма, которые имеют шесть степеней свободы и обеспечивают сохранение центра ввода инструмента без наличия избыточности приводов. Полученные расчеты дают основания утверждать, что механизмы могут использоваться для хирургических роботов. В дальнейшем рассмотрим решения задач о положениях и динамики, а также проведем экспериментальные исследования.

Работа выполнена при финансовой поддержке РФФИ (проект № 16-29-04273).

\section{ЛИТЕРАТУРА}

1. Филиппов Г.С., Глазунов В.А. Перспективы применения механизмов параллельной структуры в аддитивных технологиях изготовления центрального тела сопла турбореактивного двигателя, высокопрецизионных хирургических манипуляциях, зондовой диагностике плазменных потоков // Проблемы машиностроения и автоматизации. 2018. № 3. С. 121-128.

2. Ганиев Р.Ф., Глазунов В.А., Филиппов Г.С. Актуальные проблемы машиноведения и пути их решения. Волновые и аддитивные технологии, станкостроение, роботохирургия // Проблемы машиностроения и надежности машин. 2018. № 5. С. 16-25.

3. Ilewicz G., Wojnarowski J. Kinematics of Constant Point Mechanism of Cardiosurgical Telemanipulator // The scientific experiment is carried out in order to determine variables of joints of constant point mechanism. The motion of classical thoracoscope tools is analyzed with using of system of four digital cameras. Silesian University of Technology Gliwice Poland. 19-25 June, 2011. IMD-123.

4. Jin Y., Bi Z., Gibson R., McToal P., Morgan M., McClory C., Higgins C. Kinematic Analysis of a New Over-constrained Parallel Kinematic Machine // Its superior performance has been practically validated, but its kinematic characteristics and models have not yet been thoroughly disclosed. In this paper, the kinematic characteristics of this new ma-chine tool will be presented, which include mobility analysis, Jacobian formulation, to workspace analysis. Queens University Belfas UK Indiana University Purdue University Fort Wayne, USA. 19-25 June, 2011 A7_282

5. Nederbragt W.W., Ravani B. Kinematic Registration Using Contact Sensing // his paper presents a method for registering or determining the location of geometric elements that compose the external features of mechanical fixtures. Lawrence Livermore National Laboratory Livermore, California, Department of Mechanical and Aeronautical
Engineering University of California, Davis, California 95161 USA 19-25 June, 2011 A7-566.

6. Gosselin C., Angeles J. The Optimum Kinematic Design of a Spherical Three-Degree-of-Freedom Parallel Manipulator // Trans. ASME J. Mech., Trans, and Automat. Design, 1989. PP. 202-207.

7. Yoshikawa T. Manipulability of Robotics Mechanisms // Robotics Research: The second International Symposium, pp. 439-446, 1984.

8. Moreno H.A., Pamanes J.A. Isotropic Design of a 2 DOF Parallel Kinematic Machine with a Translational Workpiece Table // This work reveals the benefits of using a performance index, which considers the kinematics of both components together and not separately. Technical University of Madrid, Spain, Autonomous University of Coahuila Torreon, Mexico. 19-25 June, 2011 A7 278.

9. Masouleh M.T., Walter D.R., Husty M.L., Gosselin C. Forward Kinematics of the Symmetric 5-DOF Parallel Mechanisms (3R2T) Using the Linear Implicitization Algorithm // This paper investigates the formulation of the forward kinematic problem of the symmetric 5-DOF par- allel mechanisms performing three rotations and two in- dependent translations. Laval University Que'bec, Canada, University of Innsbruck Innsbruck, Austria. 19-25 June, 2011 IMD-123.

10. Fomin A., Glazunov V., Terekhova A. Development of a Novel Rotary Hexapod with a Single Drive. // Robot Design, Dynamics and Control. Proceedings of ROMANSY XXII CISM-IFToMM Symposium on Theory and Practice of Robots and Manipulators. Springer. ISBN 978-3-319-78962-0. 2018. P. 141-146.

\section{ЧЕРНЕЦОВ Роберт Александрович - аспирант, Институт машиноведения им. А. А. Благонравова РАН}

\section{ВЕЛИЕВ Евгений Ибадович -}

доктор медицинских наук, профессор, Российская медицинская академия последипломного образования

\section{ГЛАЗУНОВ Виктор Аркадьевич -}

доктор технических наук, директор, Институт машиноведения им. А. А. Благонравова РАН

\section{СКВОРцОВ Сергей Александрович -}

старший научный сотрудник, кандидат технических наук, Институт машиноведения им. А. А. Благонравова РАH

КОВАЛЕВА Наталья Львовна кандидат технических наук, старший научный сотрудник, Институт машиноведения им. А. А. Благонравова РАН 